\title{
2.1. Interaktion und Kommunikation
}

\section{Axel Schmidt}

Keywords: soziales Handeln, Interaktion, Kommunikation, Signal, Wechselseitigkeit

\section{Einleitung}

Der vorliegende Beitrag nähert sich den Begriffen Interaktion und Kommunikation aus einer soziologischen Perspektive. Einem allgemeinen Verständnis zufolge befasst sich Soziologie mit den „Arten und Weisen, wie das menschliche Leben organisiert wird“ (Joas, 2001, S. 14). Für eine solche "Organisation“ sind in einem ganz allgemeinen Sinn Prozesse der Interaktion und Kommunikation notwendig: Sie gewährleisten einen interpersonalen Austausch, was Soziales überhaupt erst entstehen lässt. In einem weiten Verständnis ist jedwede Interaktion/Kommunikation (im Folgenden mit „I“ bzw. „K“ abgekürzt) medial vermittelt. Mediensoziologie befasst sich mit der Rolle ,der Medien' innerhalb sozialer Prozesse, nimmt ihren Ausgangspunkt aber bei Prozessen der $\mathrm{I} / \mathrm{K}$, deren medial unvermittelte Formen mediale Vermittlung erst als solche profilieren (s. Schmidt in diesem Band).

Im Folgenden kann es nicht darum gehen, die aufgerufenen Grundbegriffe innerhalb einzeldisziplinärer Zugänge und damit jeweils zusammenhängender Theorietraditionen zu verorten. Vielmehr ist es das Ziel des vorliegenden Beitrags, die aufgerufenen Grundbegriffe in eine Relation zueinander zu bringen, um ihre gegenseitigen Grenzen, aber auch ihre Überschneidungen und Unschärfen zu bestimmen.

\section{Interaktion/Kommunikation als Austauschprozesse}

Gemeinsam ist den Begriffen $\mathrm{I} / \mathrm{K}$, dass sie als soziales Handeln begriffen werden ${ }^{1}$. Soziales Handeln vermag Prozesse der Aufeinander-Bezogenheit in Gang zu setzen. Ob und wie ein solches Aufeinander-Bezogensein gelingt, hängt ab von der Art des Kontakts und den kontaktermöglichenden Medien. Graumann (1972) betont, dass beide Begriffe "sich auf zwischenmenschliches Geschehen zu beziehen scheinen" (ebd., S. 1110) und damit darauf angelegt sind „der Polarisierung Individuum vs. Gesellschaft entgegenzuwirken, indem sie stärker [...] das, was zwischen Mir und dem Anderen, zwischen Individuum und Gruppe oder Gesellschaft geschieht, zu artikulieren gestatten" (ebd., S. 1111). I/K erfordert damit zumindest zwei Instanzen (A, B) sowie etwas, was ,zwischen' diesen Instanzen aufgrund ihres Verhaltens angesetzt wird. Dieses ,Zwischen' kann als gedankliche (Ko)-Orientierung (Personen richten ihr

1 Die Begriffe „Handeln“ bzw. "Soziales Handeln“ folgen der Grundlegung durch Max Weber (1980), d.h., Handeln ist ein Verhalten, das mit einem subjektiven Sinn verbunden ist, und soziales Handeln ein Handeln, das an anderen orientiert ist. 
Handeln an anderen aus), körperlich-zeichenhafte Koordinierung (gegenseitige und ,öffentliche' Ausrichtung aneinander) oder symbolischer Austausch (Fokus auf etwas Drittes (X), das sich als eine Art ,Austauschprodukt' begreifen lässt) perspektiviert werden (vgl. Esser, 2000, S. 229ff.). Die mit dem Begriff I/K bezeichneten Phänomene variieren grundsätzlich vor allem in zweierlei Hinsicht:

\section{a) Einzelne Akte oder Prozess?}

Üblicherweise werden mit Blick auf I/K-Prozesse einzelne Akte unterschieden ${ }^{2}$. Diesen wird das Potenzial zugeschrieben - entweder prospektiv/initial oder retrospektiv/ reagierend - einen Austausch herzustellen. Dabei kann der einzelne Akt entweder auf einen Austausch-Prozess angelegt sein, ohne dass aber ein solcher (vollumfänglich) zustande kommt (da etwa eine Reaktion fehlt). Oder er stellt rückwirkend durch eine entsprechende Reaktion einen Austausch her. Obwohl die Begriffe $\mathbf{I} \mathbf{K}$ also häufig von einzelnen Akten ihren Ausgang nehmen, orientieren sie sich aber immer an unterstellten Prozessen der Aufeinander-Bezogenheit ${ }^{3}$.

\section{b) Welche Prozesselemente sind notwendig?}

Hinsichtlich des zeitlichen Ablaufs kann gefragt werden, ab welchem systematischen Punkt I/K stattgefunden haben soll: Ausgehend von einem Verhalten eines Akteurs (A) bzw. einer Wahrnehmung/Reaktion eines Akteurs (B) lassen sich prinzipiell folgende systematische Punkte ansetzen:

- Ist A (bzw. dessen Handlung) für andere (B) perzeptiv zugänglich (Situation)?

- Nimmt B (die Handlung von) A wahr (Wahrnehmung/Aufmerksamkeit)?

- Nimmt A wahr, dass B ihn wahrnimmt und umgekehrt (reflexive Wahrnehmung)

- Versteht B das Verhalten von A als Mitteilung (Kundgabehandeln)?

- Versteht B die gesetzten Zeichen von A (Symbolverwendung/Decodierung)?

- Versteht B, was A damit von ihm wollte (Intention/Meinen)?

- Reagiert B darauf (Reaktion)?

- Reagiert B ,sinnentsprechend“ (Responsivität/Qualität der Reaktion)?

- Und umgekehrt bis zur Reaktion von A auf B (Sequenzialität)?

2 Für Reichertz (2009, S. 100 ff.) stellen Züge oder moves (Goffman, 2005, S. 94) bzw. turns (ein Terminus der Konversationsanalyse; vgl. Schegloff, 2007, S. 3 ff.) die Basiseinheiten eines Kommunikationsgeschehens dar. Siehe hierzu auch Schützeichel (2004, S. 64 ff.), der zwischen kommunikativen Handlungen (bezogen auf das Gelingen der Handlung als solcher) kommunikativen Akten (bezogen auf das erfolgreiche Initiieren eines Kommunikationsprozesses) unterscheidet.

3 Prospektiv/Initial: I/K ist darauf angelegt, Reaktionen zu erwirken. Retrospektiv: Eine Information wird als Mitteilung verstanden. 
Wenn eine Aufeinander-Bezogenheit unterstellbar ist, lässt sich zusätzlich fragen:

- Welche gemeinsame Handlung ist durchgeführt worden (Situationsdefinition) bzw. welche Verständigung ist dadurch erreicht worden (Intersubjektivität)?

- Welches Handlungsproblem ist dadurch (wie) bearbeitet worden?

Am (Nicht-) Vorhandensein obiger Elemente setzen unterschiedliche Konzepte von I/K an: Grob lassen sich Modelle unterscheiden, die I/K daran binden, dass a) das Signal ankommt (etwa Bentele $\&$ Beck, 1994), b) das Signal eine Reaktion hervorruft (hierauf heben die meisten Ansätze ab), c) das Signal als Mitteilung verstanden wird (etwa in systemtheoretischen Ansätzen oder d) das Signal sinnentsprechend verstanden wird (etwa bei Burkhart, 2002). Insbesondere an letzteres Kriterium knüpfen sich Vorstellungen von gelungener/misslungener I/K (vgl. hierzu Ungeheuer, 1987; Reichertz, 2009, S. 174ff.). Zugleich verbinden sich damit funktionale Zuschreibungen, d.h. welchen Zwecken ein solcher Austausch folgt (Gemeinsamkeit, Verständigung, Verhaltenskoordinierung, Lösung von Handlungsproblemen etc.).

Allen Konzeptionen gemeinsam ist, dass I/K-Prozesse einerseits mehr sind als soziales Handeln, da sie auf einen Austausch angelegt sind (was bei sozialem Handeln nicht zwingend ist). Andererseits aber nicht notwendigerweise wechselseitig sein müssen, da bereits das Angelegtsein auf eine Wechselwirkung ein Kriterium für Austausch sein kann. Das heißt: Dem einzelnen Akt ist vom Entwurf her die Reaktion Anderer eingeschrieben ${ }^{4}$.

Die Grenzen des I/K-Begriffs im Sinne eines (potenziellen) Austauschs werden damit einerseits von Einsamkeit/Absenz und anderseits von Flüchtigkeit des Handelns bzw. seiner Produkte markiert: So kann Verhalten, das einsam stattfindet und keine dauerhaften Produkte hervorbringt, keine Eindrücke oder Reaktionen hervorrufen. Erst Situationen (Anwesenheit bzw. perzeptiver Zugang) und/oder zeitüberdauernde Handlungsresultate ermöglichen ein an anderen orientiertes Handeln. Kurz: Voraussetzung für (potenziellen) Austausch ist Kontakt, der wiederum Prozesse gegenseitiger Orientierung in Gang zu setzen vermag.

Obwohl die Begriffe I/K häufig synonym verwendet werden (vgl. u.a. Bonfadelli, Jarren \& Siegert, 2005; Burkart, 2002; Graumann, 1972; Reichertz, 2009), finden sich in der einschlägigen Literatur auch Differenzen, um die es in den beiden nächsten Kapiteln gehen soll.

\section{Interaktion}

Kern des Interaktionsbegriffes ist die wechselseitige Aufeinander-Bezogenheit von Handlungen. Im Fokus ist, was ,zwischen' (Inter-) Handlungen (-aktionen) geschieht und damit der Prozess, der entsteht, wenn Handlungen sich aufeinander beziehen. Im Gegensatz zum Begriff der Kommunikation, in dessen Zentrum die Mittel und Inhal-

4 Vgl. hierzu Schütz (1974, S. 157). 
te des Austauschs stehen, ist der Ansatzpunkt des Interaktionsbegriffs die Koordination von Verhalten. In einem allgemeinen Sinn bedeutet Interaktion Wechselbeziehung oder Wechselwirkung (vgl. Simmel, 1995) zwischen zwei oder mehreren Elementen (Instanzen, Stoffe, Variablen, Personen etc.). Soziale Interaktion meint dagegen wechselseitig aufeinander bezogenes Handeln menschlicher Akteure. Eine entsprechende Definition findet sich bei Jäckel (2005, S. 47): „Interaktion meint im soziologischen Sinne die Wechselwirkung zwischen Handelnden. Nimmt man eine dyadische Interaktion als Bezugsrahmen, so beschreibt der Begriff den Prozess aufeinander bezogenen Handelns zweier Akteure“. Dabei setzt der Interaktionsbegriff an beobachtbaren Verhaltensbewegungen an, fokussiert also das ,öffentlich“ Zugängliche (vgl. Graumann, 1972). Da Interaktion im Wesentlichen über das formale Kriterium der Wechselseitigkeit von Verhalten bestimmt ist und ein zentraler Modus des Zustandekommens von Wechselseitigkeit Anwesenheit ist, lassen sich die drei folgenden Fragen stellen, um sich dem Interaktionsbegriff zu nähern:

\subsection{Was bedeutet Wechselseitigkeit?}

Als minimales Bestimmungselement fungiert - folgt man Graumann (1972) -, dass Menschen aufeinander einwirken. Dies kann allerdings - Luckmann (1992) folgend unterschiedliche Formen annehmen:

- Kausale, nicht sinnhafte Formen der Wechselwirkung (etwa Zusammenstöße) stellen Grenzfälle dar, da sie weder vorentworfen noch an anderen orientiert sind.

- Wechselwirkung durch Anwesenheit

- Orientierung am Beobachtet-Werden: „In gemeinsamer Reichweite befindet man sich immer in wechselseitiger und leibhaftiger Befangenheit" (ebd., S. 114). Goffman (1971 b) hat dies als "unfocused interaction" beschrieben und Wabrnehmungswabrnehmungen (vgl. Hausendorf, 2003) als einen zentralen Mechanismus solcherart Wechselwirkung herausgestellt.

- Wechselseitiges Wirken: Ist man sich bewusst, dass das eigene Handeln (etwa ein Auto einparken) beobachtet werden kann, orientiert man sich an diesem Umstand. Gleiches gilt für das Gegenüber. Obwohl es hier zu keiner wechselseitigen Orientierung kommen muss, wirken die so Handelnden doch aufeinander ein, da Mechanismen der Eindruckssteuerung greifen, wie Goffman $(1969,1971$ a) sie beschrieben hat. Zudem „könnten die gleichen oder nahezu die gleichen äußeren Vorgänge [...] auch als ,Anfrage' oder ,Antwort' verstanden werden" (Luckmann, 1992, S. 115), wodurch wechselseitig aneinander orientiertes Handeln oder „zentrierte Interaktion“ (Goffman, 1971 b, S. 84ff.) zustande kommt, die sich durch folgende Merkmale auszeichnet:

1. Motivverschränkung ist ein "Wirken, das nicht nur irgendwie wechselseitig aufeinander bezogen ist, sondern von den Entwürfen her aufeinander gerichtet ist [...]" (Luckmann, 1992, S. 116). Was in diesem Prozess, Wirkung' entfaltet, ist demnach die spezifische Form der wechselseitig unterstellten Motive, die darin besteht, dass der Entwurf und das damit zusam- 
menhängende Verhalten einer ersten Person zum Weil-Motiv einer zweiten (ich antworte, weil ich gefragt worden bin) und umgekehrt das antizipierte (gewünschte) Verhalten dieser zweiten Person zum Um-zu-Motiv der ersten wird (ich frage, um eine Antwort zu bekommen). Ist eine solche „ungebrochene Verkettung der Motive“ (ebd., S. 117) in Gang gekommen, sind jedem Handlungszug sowohl weil-motivische (verursacht/ kausal; retrospektiv) als auch um-zu-motivische (verursachend/teleologisch; prospektiv) Aspekte eingeschrieben. Diese Struktur einer retrospektiv-prospektiven Relevanz jedes interaktiven Handlungszugs ist paradigmatisch für Interaktionsprozesse.

2. Doppelte Kontingenz (Luhmann, 1984, S. 148ff.; Schneider, 2005, S. 110ff.) meint verschränkte Zukunftsoffenheit in zweifacher Hinsicht, da die Gesamthandlung an zwei Stellen (ego und alter) fortlaufend kontingenten Sinnselektionen unterworfen ist, mit denen die jeweils nächste Sinnselektion „zu rechnen“ hat, ohne diesen Prozess still stellen oder revidieren zu können (beide Versuche stellen bereits nächste Sinnselektionen innerhalb dieses Prozesses dar und schreiben ihn entsprechend fort).

3. Intersubjektivität: Durch den Mechanismus der Motivverschränkung wird das Problem der doppelten Kontingenz so bearbeitet, dass der Aufbau von Erwartungsstrukturen ermöglicht wird. Grundlage ist das Durchlaufen eines Aushandlungsprozesses: In der dritten Sequenzposition entsteht eine Reaktion (C) auf die Reaktion (B) der eigenen Aktion (A), was als ein Verstehen des vorangegangenen Verstehens (=die Reaktion auf die Aktion) begriffen werden kann und wodurch die Behandlung (B) der durch die initiale Aktion (A) übernommenen Verpflichtungen bzw. Folgeerwartungen eine Evaluation durch den Initiator erhält $(C)^{5}$.

\subsection{Wie kommt Wechselseitigkeit zustande?}

Wechselseitigkeit setzt voraus, dass Menschen einen Kontakt zueinander haben, d.h., dass das Handeln nicht nur an anderen orientiert ist, sondern auch wahrgenommen werden kann. Da sich Interaktionen als Episoden vollziehen (Goffman, 1977, S. 274ff.), lässt sich nach ihrem Beginn fragen. Luckmann (1992, S. 110) unterscheidet symmetrische von asymmetrischen Fällen:

a) Asymmetrisch: Wenn ein auf Wechselseitigkeit angelegtes Handeln einseitig bleibt (etwa ungehörtes Rufen) oder umgekehrt ein auf Einseitigkeit angelegtes Verhalten wechselseitig wird (etwa ein entlarvter Lauschangriff).

b) Symmetrisch: Wenn Entwurf und Vollzug übereinstimmen, also ein auf Wechselseitigkeit angelegtes Handeln sich auch wechselseitig vollzieht.

5 Zur systematischen Bedeutung der dritten Sequenzposition vgl. grundlegend Schegloff (1997) sowie Deppermann (2001, S. $77 \mathrm{ff}$. und 2008). 
Auf der Grundlage, dass soziale Handlungen einerseits sowohl auf Einseitigkeit als auch auf Wechselseitigkeit beruhen können bzw. angelegt sein können und andererseits sowohl mittelbar als auch unmittelbar erfolgen können, formuliert Luckmann (1992) des Weiteren vier „Hauptformen gesellschaftlichen Handelns“ (ebd., S. 110 eig. Herv.), die sich aus folgender Kreuztabelle ergeben:

Kontakt

Richtung

einseitig

wechselseitig unmittelbar

Taschendiebstahl

Face-to-face-Gespräche mittelbar

Fernsehen Graffiti

Telefon, Briefkontakt

Hieran wird deutlich, dass auch auf Einseitigkeit angelegtes soziales Handeln, eine Wirkung anstrebt und daher potenziell immer auch Gefahr läuft, in Wechselseitigkeit umzuschlagen ${ }^{6}$. Ist ein soziales Handeln wechselseitig, kann es mittelbar oder unmittelbar erfolgen sowie - wenn Letzteres der Fall ist - Prozesse zentrierter oder unzentrierter Interaktion hervorrufen. Während mittelbare Wechselseitigkeit gesondert hergestellt werden muss (etwa mittels des Gebrauchs eines technischen Mediums), wird unmittelbare Wechselseitigkeit bereits durch Anwesenheit erzwungen.

\subsection{Welche Rolle spielt Anwesenheit?}

Wechselseitigkeit als Grundbedingung von Interaktion setzt Kontakt voraus, welcher zunächst auf Anwesenheit beruhen kann ${ }^{7}$. Wenn dies nicht der Fall ist, kommt es zu mittelbaren Formen der Wechselseitigkeit, die gesondert arrangiert werden müssen. Interaktion lässt sich daher grundsätzlich von zwei unterschiedlichen Seiten betrachten, nämlich einmal als Folge von Aktionsformen (Interaktion kommt zustande durch auf andere bezugnehmende Verhaltensweisen), das andere Mal als Folge von Kontaktformen (Interaktion kommt zustande durch Kopräsenz, die eine Orientierung an anderen erzwingt). Die besondere Rolle der Anwesenheit im Sinne unfokussierter Interaktion liegt daher darin, dass sie nicht nur selbst rudimentäre Formen der Koorientierung (oder einen "interaction tonus" nach Goffman, 1971 b, S. 36) forciert, sondern zudem als ,Milieu' verstanden werden kann, das wechselseitige Interaktionen begünstigt ${ }^{8}$. Der Interaktionsbegriff lässt sich daher als zweiseitiges Konstrukt begreifen (vgl. Neuberger, 2007), das entweder in aktionaler Hinsicht auf kumulative Wechselseitigkeit oder in situationeller Hinsicht auf Kopräsenz abhebt:

6 Daher kann ein Verhalten auch dann interaktiv werden, wenn es nicht als Interaktion intendiert war (vgl. Posner, 1985, S. 244).

7 In dieser, „positivistischen“ (Knoblauch, 1995, S. 68) Weise fasst etwa Goffman den Begriff der Situation: „Situationen entstehen, wenn gegenseitig beobachtet wird, sie vergehen, wenn die zweitletzte Person den Schauplatz verlässt “ (Goffman, 1971 b, S. 29).

8 Dies bezeichnet Meyrowitz (1990 a) in Anlehnung an Goffman (1971 b, S. 36) als "ready to come into play" (S. 79). Multimodal orientierte Detailstudien zur Herstellung fokussierter Interaktion finden sich in Mondada \& Schmitt (2010). 
Aktional: Interaktion als kumulative Wechselseitigkeit

In dieser Hinsicht bedeutet I eine aneinander orientierte Abfolge von Handlungen (Aktion/Reaktion usw.), also „die ,aktiv-reaktive' Komponente des interpersonalen Geschehens" (Graumann, 1972, S. 1112). In den Vordergrund rückt damit nicht, was getan wird, sondern dass dies wechselseitig geschieht und dass - wie Neuberger (2007) unter Rekurs auf Rafaeli (1988) betont - Folgehandlungen nicht nur auf vorhergehende Handlungen Bezug nehmen, sondern sich auch auf die Relation beziehen, in der vorangehende Handlungen zueinander standen. Somit ist weniger die Abfolge als solche, als vielmehr die die dadurch erreichte sukzessive Kumulation von Sinn und damit die Ausbildung einer Fallstruktur (vgl. Oevermann, 2000, S. 69ff.) entscheidend. Prototypisch wird dies an der von Mead (1995) beschriebenen triadischen Gestenkommunikation greifbar: Ein erstes Verhalten (1) steckt einem zweiten Verhalten (2) durch das ihm innewohnende Ankündigungs- und Aufforderungspotenzial einen Rahmen, wodurch bereits das erste Verhalten auf eine angestrebte Gesamthandlung (3) verweist ${ }^{9}$. Demzufolge lässt sich die Interaktionen innewohnende Struktur der wechselseitigen Anpassung auf den einzelnen Akt ausweiten ${ }^{10}$. Innerhalb eines solchen, als Sequenzstruktur (vgl. Schegloff, 1972) beschreibbaren Wechselverhältnisses bilden sich systematische Positionen heraus, die einzelne Akte mit einem ,interaktionalen Wert' versehen (vgl. Schegloff, 2007; Deppermann, 2008) ${ }^{11}$. Legt man allein diesen formalen Aspekt der Wechselseitigkeit zugrunde, lässt sich auch dann noch von Interaktion sprechen, wenn der Austausch distant (Ortsverschiedenheit) und/oder asynchron (Ungleichzeitigkeit) wird. Denn - so wird gegen einen zu engen I-Begriff eingewendet - „[es] geht Anwesenheit als Kriterium [...] über das Merkmal ,Interaktion' in der Kommunikation weit hinaus" (Neuberger, 2007, S. 37), sodass insbesondere Formen medial vermittelter interpersonaler Kommunikation (wie etwa das Telefonieren oder der Briefwechsel) als Formen von Interaktion zu begreifen sind. Dennoch findet sich in einer Reihe von Beiträgen das Kriterium der Anwesenheit als notwendige Bedingung für Interaktion (vgl. Jäckel, 1995; Hausendorf et al., 2015; Merten, 1977, S. 62 f.). Dabei sind drei Aspekte relevant: Reduzierte Symptomfülle schränkt die Ausdrucksmöglichkeiten ein (Kanalreduktion), Zeitversetzung (Asynchronizität) verhindert unmittelbare Reaktionen, und die Trennung von Produktion (Handeln) und Rezeption (Wahrnehmen) verunmöglicht einen kontinuierlichen Zugang zum Anderen.

9 Vgl. hierzu insbesondere Schneider (1994, S. 131ff. sowie 2005, S. 183 ff.), der dies unter Rückgriff auf Meads (1995, S. 53 und S. 84) eigenes Beispiel, dem Kampf-/Fluchtverhalten von Hunden, verdeutlicht.

10 Siehe hierzu im Einzelnen Schneider $(1994,2002,2005)$. Insofern geht - folgt man Oevermann et al. (1983) - die Interaktion der Aktion konstitutionslogisch voraus, weshalb es irreführend sei, überhaupt von ,Inter-Aktion' zu sprechen, da dadurch suggeriert werde, dass Interaktionen sich aus Aktionen ,zusammensetzten'.

11 Die Konversationsanalyse spricht von slots und begreift diese als strukturelle Vorgaben (structural provisions), die von Interagierenden gefüllt werden (participants' work) (vgl. Jefferson, 1972; Kallmeyer \& Schütze, 1976). 
Die besonderen Qualitäten, die über das formale Kriterium der Wechselseitigkeit hinausgehen, hängen an einer gleichen Situierung in Raum und Zeit. Interaktionen zeichnen sich in dieser Hinsicht durch Synchronizität, Symptomfülle, Simultanität und Unmittelbarkeit aus, was zusammengenommen eine besondere Form der Aufeinander-Bezogenheit hervorbringt:

- Zeitgleichheit (oder Synchronizität, vgl. Schütz, 2003) bedingt, dass Reaktionen unmittelbar und spontan erfolgen müssen, sodass Interaktionen als Schlagabtausch verlaufen (vgl. Clark, 1996). So definiert Posner (1985, S. 244): „Demnach ist ein Verhalten einer Person dann interaktiv, wenn es durch zeitlich unmittelbar vorhergehendes Verhalten einer oder mehrerer Personen beeinflusst ist und seinerseits ein zeitlich unmittelbar folgendes Verhalten dieser Personen(en) beeinflusst“. Eine Vorstufe ist erreicht, wenn Personen in wechselseitigen Sichtkontakt geraten und eine Orientierung aneinander unausweichlich wird. In Kopräsenz kann jedes (Nicht)Verhalten zu jeder Zeit als Eindruckssteuerung, metakommunikativer ,Kommentar' oder interaktiver Zug ausgelegt werden.

- Verstärkt wird dies durch die vergleichsweise uneingeschränkte Wahrnehmbarkeit menschlichen Ausdrucksverhaltens (Symptomfülle), welche als komplex verzahnte Mehrebenenstruktur (Geser, 1990) hinsichtlich ihrer Kommunikativität in besonderer Weise problematisch ist.

- Simultanität bewirkt darüber hinaus eine kontinuierliche Adaption des eigenen Verhaltens auf der Basis der Wahrnehmung des Verhaltens eines Gegenübers und umgekehrt, auch und gerade während der Produktion eigenen Handelns (vgl. Goodwin, 1981).

- Anwesenheit bedingt schließlich Unmittelbarkeit und damit körperliche Zugänglichkeit, sodass bloße Anwesenheit jederzeit in eine zentrierte Interaktion münden kann, ohne dass dies einseitig kontrollierbar wäre. Zudem stehen die "Territorien des Selbst" (Goffman, 1974, S. 54ff.) in ungleich weitreichenderer Form zur Disposition als das bei vermittelten Kontaktformen der Fall ist: "Face-to-Face communication is the most intense form of communication. Every face-to-face encounter holds the possibility of embrace or attack" (Meyrowitz, 1990 b, S. 86). Allert (2008) spricht daher von „dramatischer Präsenz". Dies gilt insbesondere für intensive, existenzielle und körpernahe Formen des interaktiven Austauschs, wie etwa Sex, Kampf oder Tanz. Interaktion ist in dieser Hinsicht nicht nur eine unausweichliche Folge situationeller Bedingungen, sondern erhält ihre besondere Qualität erst durch den Umstand der Kopräsenz und ist daher in besondere Weise daran gebunden. Diesem Umstand trägt die Begriffsprägung der Face-to-Face-Interaktion Rechnung, womit wechselseitige und kopräsente Austauschformen gemeint sind ${ }^{12}$.

12 Reichertz (2009) nennt eine Reihe weiterer Konstellationen, etwa „voice-to-ear" (S. 112) (im Falle des Telefons) oder „one-to-one“ (S. 113) (wenn der gesamte Körper und nicht bloß das Gesicht (face) relevant ist), die ebenso häufig vorkämen. Letztlich geht es aber hier nicht um konkrete Erscheinungsformen von Aufmerksamkeitszuwendungen, Adressierungen oder For- 


\section{Kommunikation}

Kommunikation als analytischer Grundbegriff einer Reihe verschiedener Wissenschaftsdisziplinen ist kaum wesensmäßig definierbar (vgl. Nöth, 2000) ${ }^{13}$. Verschiedene Fassungen des Kommunikationsbegriffs sind ohne eine theoretische Einbettung kaum nachvollziehbar. Dennoch lassen sich den einzelnen Ansätzen Kriterien entnehmen, wann von Kommunikation die Rede sein soll. Dies soll im Folgenden geschehen.

Kern des Kommunikationsbegriffs ist das Einwirken auf andere durch die Vermittlung von Information und nicht - wie im Falle des Begriffs der Interaktion - die Koordination von Verhalten. Die Art der Einwirkung auf andere ist dabei in zweierlei Hinsicht eingeschränkt, woraus sich zwei Alleinstellungsmerkmale ergeben:

\section{a) Symbolvermittlung}

Eine erste Einschränkung betrifft die Art der vermittelnden Information. Eine Einwirkung geschieht nicht energetisch oder rein sensorisch, sondern symbolvermittelt (vgl. u.a. Linke, Nussbaumer \& Portmann, 1996; Esser, 2000; Reimann, 1968). Die Vermittlung von Information wird damit an den Gebrauch von Symbolen gebunden, sodass die ,Wirkung' von einer Bedeutung ausgeht. Hiermit verbindet sich die Vorstellung eines dritten Elements, das den Prozess der Einwirkung nicht bloß vermittelt, sondern in ein System geteilter Bedeutungen übersetzt und damit $\mathrm{K}$ an Operationen wie Ausdruck und Verstehen bindet. Unter Rekurs auf eine vielfach zitierte Modellierung des K-Begriffs bei Newcomb (1953) als "A-B-X-Schema“ hebt Graumann (1972) die für K grundlegende doppelte Orientierung hervor, nämlich „zwei oder mehr Individuen [...], die in Bezug auf etwas drittes sich ,ko-orientieren'. Diese Koorientierung ist nicht nur sozial, sie bezieht immer auch Aspekte der gemeinsamen Umwelt mit ein" (ebd., S. 1123). Die symbolische Qualität von K zeichnet sich nun dadurch aus, dass nicht auf Laut- oder Verhaltensbewegungen an sich reagiert wird, sondern auf deren (geteilte) Bedeutung (grundlegend Mead, 1995).

b) Mitteilungsabsicht

Eine zweite Einschränkung betrifft die Reichweite der Bedeutungsvermittlung. Da Menschen - mit Mead (1983, S. 347) gesprochen - in einer Welt voller Bedeutungen leben, ließe sich der K-Begriff - beließe man es beim Kriterium der Symbolvermittlung - auf alles ausweiten, was Menschen mit Bedeutung versehen (haben). Soll dage-

men medialer Vermittlung resp. die Abschätzung von Häufigkeiten, sondern darum, einen bestimmten Bereich sozialen Miteinanders herauszustellen, der sich durch wechselseitige Anwesenheit und die Etablierung eines gemeinsamen Aufmerksamkeitsfokus auszeichnet. Insofern geht es um zentrierte Interaktion unter kopräsenten Bedingungen. Andere Begriffe, die auf diesen eher formalen Umstand der gleichzeitigen Anwesenheit abheben sind etwa die Vis-à-Vis-Beziehung (bei Berger \& Luckmann, 1969) oder die „Wir-Beziehung“" (bei Schütz, 2003, S. 195). Eine kriteriale Bestimmung liefert Clark (1996, S. 9 f.).

13 Häufig wird in diesem Zusammenhang auf die Arbeit von Klaus Merten (1977) mit dem Hinweis verwiesen, dass dort über 160 Definitionen zusammengetragen wurden. 
gen von $K$ im engen Sinn die Rede sein, geschieht eine Vermittlung von Bedeutung handlungsgebunden und in mitteilender Absicht, d.h., die Einwirkung auf andere ist nicht nur symbolvermittelt, sondern ist als kommunikatives Handeln zu begreifen, welches Symbole zum Zwecke der Deutung explizit setzt. K impliziert also die Vorstellung einer auf andere gerichteten Entäußerungs-Aktivität. Schütz (1974) sprach in diesem Zusammenhang von „Kundgabehandeln“ (S. 157) oder Ausdruckshandlungen, welche „zwecks Deutung [gesetzt sind]“ (S. 164). Ähnlich wie Mead betont auch Schütz, dass kommunikatives Handeln die Vorentworfenheit der Reaktion potenzieller Adressaten impliziert. Aufgrund ihres damit verbundenen Potenzials, die für das menschliche Miteinander als konstitutiv erachtete Innen-Außen-Dichotomie zu überbrücken, wird Kommunikation daher häufig als Mittel zur Herstellung von Gemeinsamkeit und Gemeinschaft begriffen ${ }^{14}$. Aufbauend hierauf lässt sich $\mathrm{K}$ als Grundlage einer "gesellschaftlichen Konstruktion von Wirklichkeit" (Berger \& Luckmann, 1969) begreifen ${ }^{15}$.

Nimmt man diese beiden Kernelemente - Symbolvermittlung und Mitteilungsabsicht - zusammen, besteht Kommunikation in und durch einen spezifisch gerichteten Prozess des Sinnsetzens auf der einen und des Sinndeutens solchen Sinns auf der anderen Seite. Grenzen ergeben sich daher

1. zu nicht symbolvermittelten Formen der Einwirkung (etwa Reaktionen auf Reize oder kausal bedingte Effekte, etwa Folgen mechanischer Einwirkungen);

2. zu inneren Prozessen oder innerem Tun, etwa Denken oder Emotionen, die keinen wahrnehmbaren Ausdruck hervorbringen, auf den reagiert werden könnte;

3. zu Informationen von Anzeichenqualität, denen keine Mitteilungsabsicht unterstellt werden kann.

Solche Grundannahmen wurden vielfach in Vorstellungen darüber überführt, welche Komponenten oder Faktoren $\mathrm{K}$ enthält, in welchen Relationen diese stehen und wie man sich den Prozess der $\mathrm{K}$ vorzustellen hat. Auf solche Modelle von Kommunikation kann hier nicht ausführlich eingegangen werden. Auf zwei, für die Begriffsbestimmung von Kommunikation relevante Aspekte sei allerdings hingewiesen:

14 Diesen Punkt heben Ansätze hervor, die $\mathrm{K}$ als grundsätzlich fallibel (grundlegend Ungeheuer, 1987) oder als unwahrscheinlich (grundlegend Luhmann, 1988) konzipieren. Grundlage hierfür ist die Annahme einer prinzipiellen Innen-Außen-Dichotomie, die menschliches Bewusstsein/ Gedanken füreinander grundsätzlich unzugänglich macht: „Die Köpfe sind undurchsichtig“ (Fuchs, 1993, S. 15 f.). Zu einer Kritik solcher Ansätze vgl. Reichertz (2009, S. 149 ff.).

15 An einem solchen Verständnis von Kommunikation knüpften insbesondere Ansätze des sogenannten ,interpretativen Paradigmas“ (Wilson, 1973) an, welche Gesellschaft als fortwährenden symbolischen Aushandlungsprozess konzipieren. In besonderer Weise hervorgehoben wird dieser Umstand aktuell in Ansätzen des sogenannten „kommunikativen Konstruktivismus“, welche unter Rekurs auf Berger \& Luckmann (1969) die konstitutive Funktion von K für Prozesse sozialer Wirklichkeitskonstruktion hervorheben (vgl. hierzu Keller, Reichertz \& Knoblauch, 2013). Insbesondere innerhalb systemtheoretischer Ansätze wird das Bestehen von Gesellschaft an $\mathrm{K}$ geknüpft, mehr noch: Soziale Systeme reproduzieren sich allein in und durch Kommunikation (vgl. Luhmann, 1997). 
a) Die meisten Modelle setzen folgende Grundkomponenten voraus:

- Instanzen (mindestens zwei: A, B);

- ein Drittes: Mitgeteiltes, Botschaft, Nachricht, Sinnselektion etc. (X).

So etwa grundlegend Nöth (2000, S. 235): „Grundbedingung für das Zustandekommen einer Kommunikation ist ein Semioseprozess, an dem ein Kommunikator, ein Zeichen bzw. eine Botschaft und ein Rezipient oder Interpret des Zeichens beteiligt sind“.

Wird - wie oben hergeleitet wurde - K als Prozess intentionaler Zeichensetzung (Kriterium: Mitteilungsabsicht) konzipiert, ergibt sich die Notwendigkeit einer weiteren Komponente:

- gerichtetes kundgebendes Handeln, durch das dieses Dritte in Hinblick auf eine Kundnahme erzeugt wird und daher im Kundgeben potenziell mit angelegt ist; Kundnahme, durch das dieses Dritte (als Kundgabe) wahrgenommen und verarbeitet wird.

Die Vorstellung, dass eine erste Instanz etwas in Hinblick auf eine zweite Instanz erzeugt, was diese wahrnehmen/verarbeiten soll, macht Fragen nach dem Prozess der Übermittlung relevant, was Bedarf für weitere Komponenten schafft (welche dann insbesondere für den Begriff der Medien relevant werden; siehe Schmidt in diesem Band):

- etwas, was dieses Dritte materialisiert bzw. wahrnehmbar macht:

- generell: wahrnehmbare Ereignisse oder Objekte;

- in semiotischer Hinsicht: Zeichenträger, Signifikant etc. (etwa Laute);

- etwas, was diesem Dritten geteilte Bedeutung verleiht: Zeichen(system), Symbol, Code etc. (z.B. Körperbewegungen als Gesten, Laute als Sprachlaute sowie damit zusammenhängende Zeichensysteme, etwa ,Körpersprache' oder Sprache);

- etwas, was dieses Dritte ,vermittelt': Kanal, Medium, Bote (z.B. bei Körperbewegungen: Licht, bei Sprachlauten: Schallwellen/Luft).

Auf einer solchen Vorstellung der Übertragung von Botschaften/Nachrichten beruhen klassische Sender/Empfänger-Modelle, die sich innerhalb verschiedenster Disziplinen finden. Prominent geworden sind etwa Ferdinand de Saussures (1967) Redekreislauf für die Sprachwissenschaft oder das informationstheoretische Modell von Claude E. Shannon und Warren Weaver (1949) für die Kommunikationswissenschaft.

\section{b) Kritik an klassischen Modellen}

Übertragungsmodelle liefern eine erste Einsicht in den Prozess der K, vor allem seiner Grundkomponenten und seiner medialen Notwendigkeiten. Allerdings - so die Kritik - werde der Prozess der K unzulässig vereinfacht.

Eine erste Kritik setzt am Konzept der Übertragung an: Insbesondere konstruktivistische Ansätze beanstanden, dass durch die Vorstellung einer Übertragung Information, Botschaft oder Bedeutung vergegenständlicht werde. Entsprechende Konzepte 
werden daher als "Container-Modelle" kritisiert: „Denn übertragen kann man nur etwa Gegenständliches. Und wenn etwas übertragen wird, dann brauchen wir einen Behälter (oder Container), in dem die Information von einem Sender zu einem Empfänger übertragen wird“ (Schmidt, 1994, S. 51). Informationen als Entitäten aufzufassen hat weitreichende Konsequenzen, etwa die irrführende Vorstellung "gleiche Botschaften müssten auch gleiche Wirkungen haben sowie der darauf gegründete Versuch, von Botschaften auf Wirkungen ,hochzurechnen'“ (ebd., S. 53). So vollzieht sich menschliche Kommunikation nicht durch die Übertragung von Nachrichten oder Informationen; vielmehr kommt $\mathrm{K}$ zustande, indem Menschen spezifische Auswablen treffen: Der Rezipient generiert Bedeutung durch eigenständige Selektionsaktivitäten aus den Strukturmerkmalen einer angebotenen Information, die nicht übertragen, sondern „sozusagen hausintern erst erzeugt“ (Rusch, 1994, S. 67) wird. Zudem bleiben solche Modelle „dadurch unvollkommen, dass die Gleichzeitigkeit des kommunikativ Aufeinander-Bezogenseins in eine Sequenz linearer Prozesse übersetzt wird“ (Graumann, 1972, S. 1120). Insgesamt konstatiert Schmidt (1994, S. 51ff.) eine Entwicklung von Modellen der Signalübertragung hin zu Modellen der Informationskonstruktion.

Eine zweite Kritik moniert die Einengung auf bewusste Handlungen und explizite, an einen Adressaten gerichtete Mitteilungen, da auf diese Weise bedeutsame Bereiche ausgespart bleiben, etwa:

- das Nonverbale oder performative Aspekte (vgl. Graumann, 1972; Nöth, 2000);

- Darstellungen, Inszenierungen und Täuschungen bzw. Beeinflussungen, die gerade nicht als Mitteilungen verstanden werden sollen (vgl. Reichertz, 2009; Hahn, 2002) ${ }^{16}$;

- kulturelle Artefakte (etwa Körpergestaltungen, Kleidung oder dinglicher Besitz), welchen insbesondere in Form von Statussymbolen und Stilen kommunikative Relevanz zukommt (Schulze, 1995).

Insgesamt - so Nöth (2000, S. $235 \mathrm{ff}$.) - gerät das vielfach als Notwendigkeit für K erachtete Kriterium der Intentionalität - insbesondere im Falle nonverbaler und visueller K - an seine Grenzen.

c) Unterschiedliche Fassungen des K-Begriffs ergeben sich daher durch unterschiedliche Umgangsweisen mit der Frage der Intentionalität. Dies betrifft zum einen die Nicht-Beobachtbarkeit von Intentionen (Intentionalitäts-Dilemma) und zum anderen die Frage, wie weit der Begriff der Intentionalität für $\mathrm{K}$ angesetzt werden soll (Intentionalitäts-Reichweite).

- Intentionalitäts-Dilemma: Obwohl es nicht möglich ist, von außen zu entscheiden, ob ein Verhalten in mitteilender Absicht erfolgt, ist es gerade diese Differenz, die einen entscheidenden Unterschied macht (sowohl bei den Akteuren selbst als auch bei wissenschaftlichen Beobachtern). Dieses Problem kann vermieden werden, indem die Entscheidung, ob ein Verhalten intentional erfolgte oder nicht, dem Kommunikati-

$16 \mathrm{Vgl}$. hierzu insbesondere die von Goffman (1981 b) beschriebenen „response cries“. 
onsprozess selbst überantwortet wird: Systemtheoretische Ansätze schlagen etwa vor, dann von $\mathrm{K} \mathrm{zu}$ sprechen, wenn eine Information als Mitteilung verstanden wurde (vgl. Luhmann, 1984, S. 191ff.). Intentionalität wird als „kommunikative Reduktionsform subjektiven Sinns“ (Schneider, 2004, S. 294) begriffen. Auf diese Weise fungieren Intentionalitätszuschreibungen als empirisches Kriterium, um $\mathrm{K}$ zu fassen, was letztlich durch Folgehandlungen gegeben ist ${ }^{17} . \mathrm{K}$ lässt sich demzufolge als Aushandlungsprodukt auf Gegenstandsebene konzipieren, sodass eine Differenzierung von

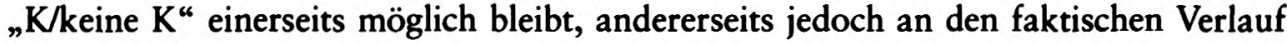
des Kommunikationsprozesses selbst gebunden wird.

Intentionalitäts-Reichweite

- Weit: Allem Verhalten, das sinnhaft ist sowie allen Objekten, die durch sinnhaftes menschliches Tun zustande kamen (=kulturelle Artefakte), kann eine kommunikative Funktion unterstellt werden; $\mathrm{K}$ geht auf diese Weise in der Vorstellung einer grundsätzlich sinnstrukturierten Sozialwelt auf;

- Eng: Erst wenn ein Verhalten, nicht nur sinnhaft ist, sondern sich selbst als Mitteilung ausweist, kann von $\mathrm{K}$ gesprochen werden. Dies impliziert doppelte Intentionalität (Einwirkung in Form einer Mitteilung) und doppelte Inferenzialität (Schluss auf Sache und Person). Eine solche Position vertritt etwas Posner (1985), wenn er $K$ wie folgt fasst: „Der Sender [will] den Adressaten mit seinem Verhalten nicht bloß dazu bewegen, dass er einen bestimmten Schluss vollzieht, sondern auch dass er diesen Schluss aufgrund der Annahme vollzieht, dass der Sender ihn dazu bewegen will“ (ebd., S. 243). Typischerweise geschieht dies durch den Gebrauch von Sprache, der als explizites Kommunikationsmittel eine solche mitteilungsintentionale Infrastruktur eingeschrieben ist (vgl. Luhmann, 1997, S. 205ff.).

- Vermittelnde Position: Kommunikativ ist ein Verhalten auch dann, wenn es hervorgebracht wurde, um auf andere einzuwirken, ohne dies allerdings explizit als Mitteilung zu rahmen. Rusch (1994, S. 66) spricht von einer „Orientierungsintention aufseiten eines kommunikativ Handelnden“. In ritueller Hinsicht handelt es sich, mit Goffman (1971 a) gesprochen, um Prozesse der „Eindrucksmanipulation“.

Nachdem I und K zunächst allgemein als Austauschprozesse eingeführt (Kapitel 1) und nachfolgend als fokussierend auf wechselseitige Verhaltenskoordination bzw. symbolisches Mitteilungshandeln getrennt voneinander erläutert wurden (Kapitel 3 und 4), sollen im abschließenden Kapitel beide Begriffe in Hinblick auf ihre Gemeinsamkeiten und Differenzen nochmals verglichen werden.

17 Insbesondere Schneider (2004) hat systemtheoretische, objektiv hermeneutische und konversationsanalytische Ansätze unter einer solchen, theorieverbindenden Perspektive diskutiert. 
Die obigen Ausführungen haben $\mathrm{K}$ und I als an anderen orientiertes Verhalten gefasst, in welchem sich Sinnsetzung und -deutung sowie die Verfolgung praktischer Zwecke verschränken. Erst dieser praktische Sinnzusammenhang macht Kommunikation verstehbar. Daher betont Reichertz (2009) unter Rekurs auf Habermas (1981) dass „das Verstehen einer symbolischen Äußerung grundsätzlich die Teilnahme an einem Prozess der Verständigung [erfordert]" (ebd., S. 165.). Geschieht dies in Bezug aufeinander, ist Kommunikation Handlungskoordination mithilfe von Symbolen in praktischen Situationen und fällt so mit dem Begriff der Interaktion zusammen. Sollen beide Begriffe daher different gefasst werden, lässt sich auf oben genannte Aspekte im Sinne von Alleinstellungsmerkmalen abheben:

- Definition Interaktion: Interaktion bezeichnet Prozesse sozialer Aufeinander-Bezogenheit, die durch Anwesenheit oder mediale Vermittlung wechselseitig sind; Wechselseitigkeit im engeren Sinne bedeutet eine Verschränkung von Motiven;

- Definition Kommunikation: Kommunikation bezeichnet Prozesse sozialer Aufeinander-Bezogenheit, wobei mindestens ein Einzelakt sich durch (zugeschriebene) Mitteilungsabsicht und damit zusammenhängender Zeichenverwendung auszeichnet.

In entsprechenden I/K-differenzierenden Fassungen der Begriffe fungieren die Kriterien Wechselseitigkeit bzw. Anwesenheit (I) sowie Mitteilungsintention und Zeichenverwendung $(\mathrm{K})$ als Alleinstellungsmerkmale. Daher lassen sich interaktive aber nicht kommunikative (fehlende Mitteilungsabsicht/fehlende Zeichenverwendung) von kommunikativen aber nicht interaktiven (fehlende Anwesenheit/fehlende Wechselseitigkeit) Prozessen unterscheiden ${ }^{18}$ :

- Prozesse der Aufeinander-Bezogenheit, die nicht in Kopräsenz verlaufen und medial keine Wechselseitigkeit ermöglichen (etwa Massenkommunikation wie das Fernsehen) bzw. empirisch keine Wechselseitigkeit realisieren (etwa ungehörte Rufe, unkommentierte Facebook-Posts oder Graffiti) sind keine Interaktion, wohl aber Kommunikation;

- Prozesse der Aufeinander-Bezogenheit, die keine Zeichenverwendung in Mitteilungsabsicht kennzeichnet, sind keine Kommunikation, wohl aber Interaktion (etwa gemeinsames Arbeiten, Kampf, Sex oder Tanz.)

Die bisherigen Ausführungen haben allerdings nun schon angedeutet, dass sich solche, nach eindeutigen Merkmalen strebende Begriffsfestlegungen wiederum problematisieren lassen, wodurch es zu einer Erweiterung und gegenseitigen (Wieder-) Annäherung der Begriffe kommt:

18 Vgl. hierzu insbesondere Posner (1985), der die vier möglichen Fälle (1. interaktiv/kommunikativ; 2. interaktiv/nicht kommunikativ; 3. nicht interaktiv/kommunikativ; 4. nicht kommunikativ/nicht interaktiv) ausführlich analysiert und anhand von Beispielen veranschaulicht. 
Erweiterungen des I-Begriffs: Von der Face-to-Face-Interaktion zu mittelbaren Formen:

- Erste Erweiterung: Legt man das formale Kriterium der Zwei- oder Dreizügigkeit zugrunde, ist auch jede medial vermittelte $K$ als Interaktion zu verstehen, solange die Partner wechselseitig aufeinander Bezug nehmen (etwa Telefon oder Chat).

- Zweite Erweiterung: Selbst wenn eine solche Wechselseitigkeit technisch nicht möglich ist, (wie etwa bei massenmedialen Kommunikationsformen) kann aufgrund von Mitteilungsintentionalität (etwa bei Fernsehsendungen) davon ausgegangen werden, dass a) eine Antwort (in welcher Form auch immer), gesucht ${ }^{6}$ wird, was sich wiederum in das Produkt einschreibt (vgl. Wulff, 1993), und zudem b) ein Austausch angestrebt wird (etwa in Form eines Warentauschs, vgl. Charlton, 2001). Beides verleiht solchen Austauschformen interaktive Qualität, da nicht nur erste Züge (hier: Sendungen) an einer ,Antwort' orientiert sind, sondern auch ein dreizügiger Austausch (Angebot/Annahme/Weitersenden) in formaler und vermittelter Form zustande kommt. Thompson (1995) hat daher im Falle massenmedialer Kommunikation von „Mediated Quasi-Interaction“ (S. 82ff.) gesprochen.

Erweiterungen des K-Begriffs: Von Mitteilungen zu Sinnstrukturiertheit und -zuschreibung

- Erste Erweiterung: kommunikative Kontexte:

Verhaltensweisen erhalten einen ,kommunikativen Wert', sobald sie auf kommunikative Kontexte bezogen werden. Sie sind dann - so Reichertz (2009, S. 100) - „eine Bewegung, die im Spiel Bedeutung und Folgen hat". Das gilt zunächst offensichtlich für in zentrierte Interaktionen eingebettete instrumentelle Handlungen (etwa wenn auf eine sprachliche Aufforderung hin wortlos ein Fenster geschlossen wird). Aber auch nicht in zentrierte Interaktionen eingebettetes Tun entfaltet unter Beobachtung kommunikative Qualität ${ }^{19}$. Gleiches gilt für den gesamten Bereich des verhaltensförmigen Selbstausdrucks ${ }^{20}$. Schließlich können auch nicht intentionale Ausdrucksformen (Verhalten, etwa Augenzucken) prozessbezogen als $\mathrm{K}$ verstanden werden.

- Zweite Erweiterung: Gegenstände und kulturalisierte Natur:

Alle kulturellen Artefakte sind Produkte sinnhaften Handelns, haben daher Zeichenqualität und somit kommunikative Bedeutung (etwa im Goffman'schen Sinn als „Requisiten“, „Fassaden“ etc.) (vgl. auch Reichertz, 2009, S. 143ff.). Dies gilt insbesondere für alle Formen des Selbstausdrucks, die sich in materiellen Handlungsresultaten niederschlagen (Kleidung, Styling etc.). Auch ,natürliche' Ereignisse, Gegenstände und Zustände haben im Sinne einer kulturell überformten Natur eine kulturelle Bedeutung.

19 Watzlawick, Beavin \& Jackson (1969, S. 53) prägten hierfür die Formel „man kann nicht nicht kommunizieren (siehe hierzu kritisch würdigend auch Reichertz (2009, S. 124ff.)).

20 Reichertz (2009, S. 118) spricht von „kommunikativem Tun", Knoblauch von "minimal sinnhaftem Ausdruck“ (2013, S. 31). 
Die Grenze von I gegenüber $K$ ist über das Kriterium der Wechselseitigkeit vergleichsweise formal (Sequenzialität) und empirisch bestimmbar (ob und welche Reaktionen auf ein Verhalten stattfinden, lässt sich beobachten). Liegt grundsätzlich ein Wechselbezug vor, geht es um die Qualität dieses Wechselbezugs. Dies wird an drei Aspekten festgemacht:

1. Welche Handlungen bzw. Handlungsresultate können als interaktive Züge gewertet werden (Verhaltensbewegungen jedweder Art, Gesten, sprachliche Äußerungen, schriftliche Fixate bis hin zu ,Zügen' innerhalb eines mittelbaren Warentauschs)?

2. Welche zeitliche Spanne liegt zwischen den einzelnen Zügen (synchron vs. asynchron)?

3. Sind Reaktionen anderer bereits während der Produktion eigener Handlungen wahrnehmbar (unmittelbar vs. mittelbar)?

4. Welche systematischen Schritte müssen mindestens erfolgen? (Ein-, Zwei- oder Dreizügigkeit).

Die Grenze von $K$ gegenüber $I$ ist dagegen über die Kriterien Mitteilungsabsicht und Symbolgebrauch auf ein mentales (und damit nicht beobachtbares) Kriterium verwie$\operatorname{sen}^{21}$. Das Grundproblem besteht nun aber nicht nur darin, dass jedes Verhalten Mitteilungscharakter haben kann, aber nicht muss, sondern zudem, dass ein solcher Mitteilungscharakter offen mitkommuniziert werden kann oder nicht. Das führt dazu, dass durch ein Verhalten

1. etwas mitgeteilt werden kann, was offen als Mitteilung auftritt (prominent etwa durch Sprechen) ${ }^{22}$;

2. etwas (mit) zum Ausdruck kommt, was nicht als Mitteilung gedacht ist und daher zwar als anzeichenhafte Information (Symptom) jedoch nicht als Mitteilung gelesen wird (etwa körperliche Distanzen oder Körperhaltungen);

3. etwas, was nur (mit) zum Ausdruck zu kommen scheint, jedoch absichtlich hervorgebracht werden kann, ohne dies offen zu markieren ${ }^{23}$;

4. etwas gezeigt werden kann (etwa eine bestimmte Frisur), was eine bestimmte kulturelle Bedeutung hat und dessen Sinn zwar mit dem Träger (im Sinne von Identi-

21 Die sich hinter dem Kriterium „Symbolgebrauch“ verbergende Frage, ob ein beobachtbares Ereignis ein Zeichen oder ein Anzeichen ist, verweist letztlich wiederum auf Intentionalität.

22 Sprechen ist kaum in nicht mitteilungsintentionaler Weise zu gebrauchen, sodass allein die Verwendung von Sprache eine Mitteilungsintention metakommuniziert (vgl. hierzu Luhmann, 1997, S. 205ff.). Bei körperlichen Verhaltensbewegungen bleibt uneindeutig, ob etwas mitgeteilt wird oder nicht (Vgl. Geser, 1990).

23 Goffman (1977, S. 98ff.) konzipierte dies als Simulation oder Täuschung. Hintergrund ist die Vorstellung einer geplanten Eindruckssteuerung, die auf Grundlage der Eigendynamik menschlichen Ausdrucksverhaltens auf diverse Strategien der (Dis-)Simulation zurückgreift (siehe insbesondere Goffman, 1969 sowie Willmes, 1997), um entsprechende Eindrücke zu erwirken oder aufrechtzuerhalten. Dabei - so Goffman (1977) - sind insbesondere jene Ausdrucksdimensionen, die als besonders inszenierungsresistent gelten, zugleich auch jene, die sich besonders gut zum Täuschen eignen (ebd., S. 278). 
tätsrelevanz) verbunden wird, diesem jedoch nicht im Sinne eines kommunikativen Akts, der eine Reaktion verlangt, ausgelegt wird ${ }^{24}$.

\section{Fazit}

Eine Konsequenz der geschilderten Komplexität kommunikativer Verhältnisse in Interaktionen ist, dass beide Begriffe häufig so konzipiert werden, dass sie ineinander aufgehen. Zentrales Argument ist, dass alles, was Menschen im Beisein anderer Menschen tun bzw. an Artefakten ,mitbringen' (etwa Kleidung), die Qualität hat, ,informativ' auf andere einzuwirken. Graumann (1972) argumentiert in diesem Zusammenhang wie folgt: „Im Grunde in allen Interaktionskonzepten impliziert, lediglich kaum thematisch, ist, dass alle [...] Formen sozialer Wechselwirkung nur dadurch möglich sind, dass Menschen voneinander Kenntnis nehmen, Interaktion also immer auch Austausch von Information ist. Dieser aber wird in der Regel als Kommunikation bezeichnet“ (Graumann 1972, S. 1117). Und an anderer Stelle heißt es: „Wenn Interaktion immer so viel bedeutet, dass das Verhalten des einen Auswirkungen auf das Verhalten des anderen hat, dann können solche Wirkungen nur auf zweierlei Wegen vermittelt werden: entweder durch die Übertragung von Energie oder von Information" (ebd., S. 1179). Jedoch auch Formen der Energieübertragung (etwa Kampf, Sexualität etc.) haben ihre Bedeutung für die Interagierenden ${ }^{25}$. Daher: „Im Folgenden werden wir davon ausgehen, dass alles Verhalten in interpersonalen Situationen Mitteilungscharakter hat, gleich ob diese Interaktion absichtlich oder unabsichtlich von einem oder beiden Interagierenden in Gang gebracht und gehalten wird" (ebd., S. 1181). Bonfadelli et al. (2005) konstatieren aus heutiger Perspektive daher: „ohne Kommunikation keine Interaktion - ohne Interaktion keine Kommunikation" (ebd., S. 76), denn: „Die beiden Begriffe bezeichnen [...] nicht unterschiedliche Dinge, sondern sind wie zwei Seiten einer Münze: Es sind je andere Sichtweisen oder Perspektiven desselben Phänomens: Mit Interaktion bezieht man sich mehr auf die Beziehungsebene zwischen zwei Personen A und B, mit Kommunikation meint man die Inhaltsebene" (ebd., S. 76). Ähnlich argumentiert auch Reichertz (2009), wenn er vorschlägt, den Begriff der Kommunikation zu vermeiden und stattdessen von „kommunikativem Tun" (ebd., S. 118) zu sprechen.

Solche Konzeptionen von I/K lassen deutlich werden, dass beide Begriffe im aufeinander bezogenen Setzen und/oder Deuten von Sinn im Rahmen praktischer Handlungsvollzüge konvergieren:

Einerseits kann mit Blick auf Interaktion jeder menschliche Ausdruck immer auf seine (impliziten) Mitteilungsanteile hin befragt werden, wobei - wie oben argumentiert

24 Siehe hierzu Reichertz (2009, S. 143ff.) sowie Hebdige (1998), der subkulturelle Stile als Formen absichtlicher Kommunikation diskutiert.

25 „Try to think of an instance of human interaction, [...], that does not include message sending. [...]. You will probably not be able to think of such instance unless one of the persons is unconscious" (Newcomb, Turner \& Converse, 1965, S. 189 zitiert nach Graumann, 1972, S. 1180). 
wurde - die Grenze zwischen absichtsvoll Mittgeteiltem und bloß anzeichenhaft Interpretierbarem fließend bleiben muss, da diese Grenze aufgrund der Intransparenz des Fremdpsychischen notorisch beobachter- und damit interpretationsabhängig bleibt und daher immer wieder neu auszuhandeln ist. Nimmt man nun die Perspektive der Kommunikation ein, ergibt sich hieraus ein erweiterter Gegenstandbereich, der gerade auch das als Kommunikation begriffen wissen möchte, was nicht als explizite (vor allem: sprachliche) Mitteilung gilt (vgl. Goffman, 1969 S. 6ff.). Hieraus ergibt sich - wenn man so möchte: programmatisch -, dass Kommunikation auf das Feld der Interaktion auszuweiten ist ${ }^{26}$. In Rechnung zu stellen ist dann allerdings die Rabmungstätigkeit in Interaktionen selbst, also der Umstand, dass Akteure durchaus unterscheiden, ob ein Verhalten anderer mehr oder weniger explizit als Mitteilung erfolgt bzw. erfolgen soll (Hahn, 2002). Hieraus ergibt sich die Notwendigkeit sinntypologisch zwischen verschiedenen Ebenen menschlichen Verhaltens $z u$ differenzieren, da es ein Unterschied macht, als was ein wahrnehmbares Verhalten hinsichtlich seiner Hervorbringensbedingungen von den Akteuren selbst behandelt wird. So existiert eine Reihe von Begriffsprägungen, die diesem Umstand Rechnung tragen. Unter anderem etwa:

- Ausdruck, den sich jemand selbst gibt (signs given)/Ausdruck, den er ausstrahlt (signs given off) (Goffman, 1969, S. 6); Letzteres bezeichnet er auch als Ausdrucksinformationen (Goffman, $1981 \mathrm{a}$ );

- zum Ausdruck gebracht, kommt zum Ausdruck (Schütz, 1974, S. 31) oder Ausdruckhandlung vs. Ausdrucksbewegung (Schütz, 1974, S. 164);

- Zeichen/Anzeichen (Schütz \& Luckmann, 1984, S. 178ff.; zusammenfassend Knoblauch, 1995, S. 45 f.);

- Ausdruck, Darstellung, Inszenierung, Performance, Präsentation (Willems, 2012, S. 328ff.);

- Information vs. Mitteilung (Luhmann, 1984, S. 191ff.).

Deutlich wird daran, dass unter Bedingungen von Anwesenheit von einer „kommunikativen Mehrebenstruktur" (Geser, 1990) auszugehen ist, was es unmöglich macht, die Grenzen von K (in einem engeren Sinn) eindeutig festzulegen. Möchte man aber den K-Begriff insoweit einschränken, als dass zumindest das Verhalten sich Unbeobachtet-Fühlender nicht auch als $\mathrm{K}$ bezeichnet wird, dann kommt als Grenzkriterium nur Anwesenheit infrage. Das hieße umgekehrt, dass Verhalten, sobald es in Gegenwart anderer stattfindet, per se kommunikativ ist ${ }^{27}$. Mit anderen Worten: Anwesenheit macht Verhaltensausdruck kommunikativ oder kurz: Anwesenheit „kommunika-

26 Hierfür plädiert Reichertz, 2009.

27 So argumentiert grundsätzlich Reichertz (2009, S. 132): „Auch der Jäger, der alleine auf das Reh ansitzt, kommuniziert nicht, solange ihn kein anderer Mensch wahrnimmt. Wird er aber von einem anderen, z.B. einem Wilderer, wahrgenommen, dann kommuniziert er: das ist zwar kommunikatives Tun und kein kommunikatives Handeln, aber dennoch zweifellos Kommunikation". 
tivisiert" Verhalten. Das hat nun wiederum den Effekt, dass $\mathrm{K}$ an ein für I zentrales Kriterium, nämlich die Anwesenheit, gebunden wird.

Andererseits kann mit Blick auf Kommunikation behauptet werden, dass jeder kommunikative Akt letztlich eine Reaktion (bzw. zumindest eine Wirkung) sucht, also als Kundgabehandeln an etwaigem (potenziellem/zukünftigem) Kundnehmenden und entsprechenden Folgen orientiert ist. Daher ist auch einseitige (Massen-)Kommunikation letztlich - wenn nicht im engen Sinne interaktiv - so doch zumindest antwortsuchend (vgl. Scannell, 1991) ${ }^{28}$. Entsprechende Kommunikate sind daher auf Kontakt angelegt, und diese Kontaktsuche ist ihnen auch eingeschrieben ${ }^{29}$.

Das Verhältnis der beiden Begriffe - so durfte deutlich geworden sein - lässt sich weder als ein sich wechselseitig gänzlich ausschließendes noch als ein vollständig synonymes fassen. Die Begriffe überschneiden sich, haben aber dennoch Bereiche ausgebildet, die eher dem einen oder dem anderen Begriff zugeschlagen werden, wobei Begriffsöffnungen - wie sie oben skizziert wurden - solche Bereiche dem jeweils anderen Begriff wiederum annähern. Dies sei anhand folgender Übersicht veranschaulicht.

28 Am deutlichsten wird dies durch parasoziale Interaktion (vgl. Horton \& Wohl, 1956), bei der sogenannte Personae (=Personen, die fernsehspezifische Rollen begleiten, etwa Moderatoren/ Moderatorinnen) das Publikum direkt adressieren, indem sie in die Kamera sprechen und schauen (siehe Hausendorf, 2001).

29 Vgl. Wulff (1993), der im Falle des Fernsehens von Phatikalität bzw. Parasozialität spricht. Siehe auch Schmidt (2011), der Fernsehkommunikation als "gekappte Wechselseitigkeit" und "halbierte Responsivität" rekonstruiert (ebd., S. 222ff.). 


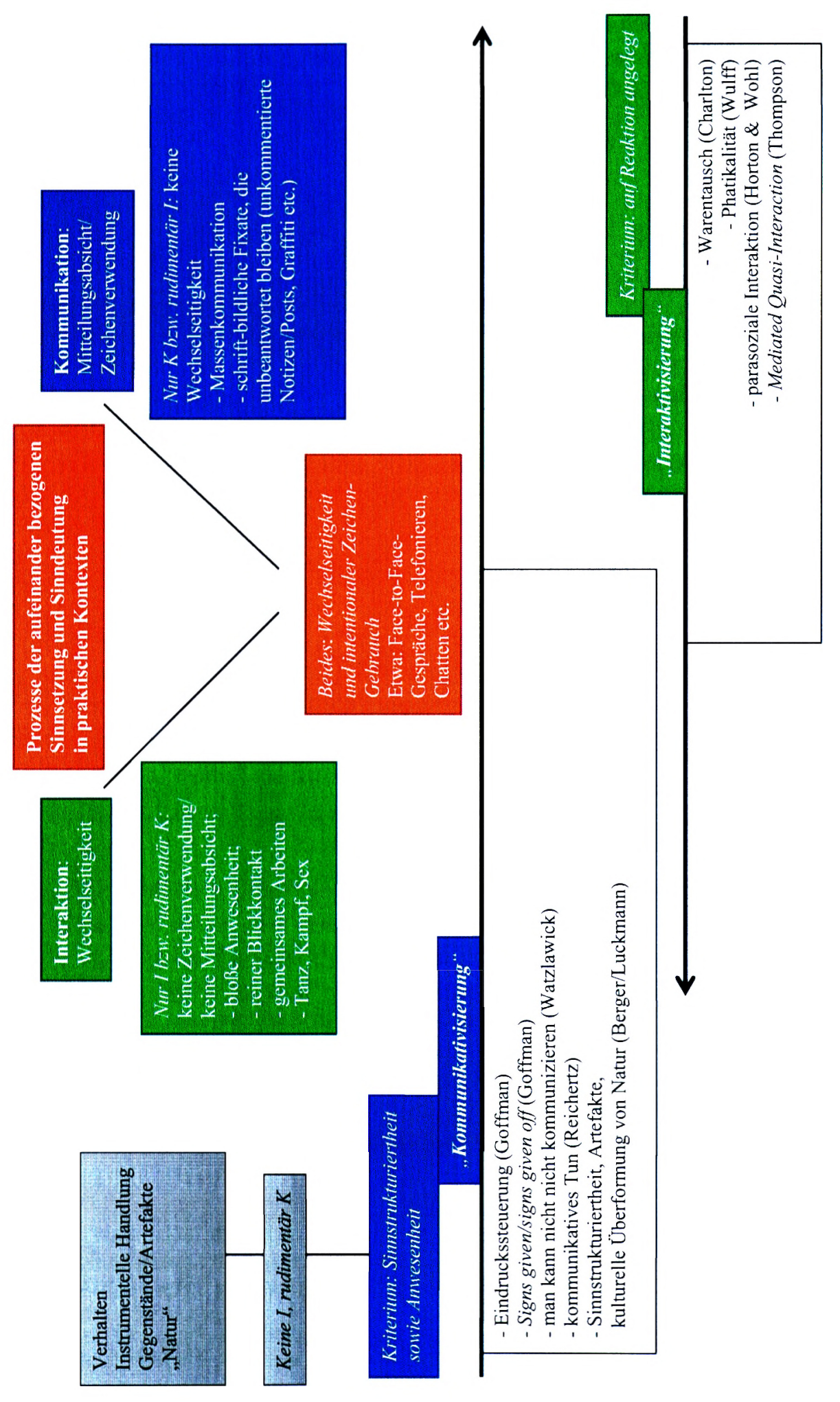

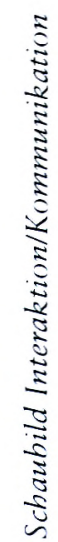




\section{Literaturempfehlungen}

Geser, Hans (1990). Die kommunikative Mehrebenenstruktur elementarer Interaktionen. Kölner Zeitschrift für Soziologie und Sozialpsychologie, 42, (2), 207-231.

Goffman, Erving (1971). Verhalten in sozialen Situationen: Strukturen und Regeln der Interaktion im öffentlichen Raum. Gütersloh: Bertelsmann.

Knoblauch, Hubert (1995). Kommunikationskultur: Die kommunikative Konstruktion kultureller Kontexte. Berlin/New York: de Gruyter.

Luckmann, Thomas (1992). Theorie des sozialen Handelns. Berlin/New York: de Gruyter.

Reichertz, Jo (2009). Kommunikationsmacht. Was ist Kommunikation und was vermag sie? Und wesbalb vermag sie das? Wiesbaden: VS.

\section{Literatur}

Allert, Tilman (2008). Grundformen sozialen Lebens: Eine Einfübrung in die Soziologie. Original-Vorträge. Tübingen/Müllheim: Auditorium Netzwerk.

Bentele, Günter \& Beck, Klaus (1994). Information - Kommunikation - Massenkommunikation. In O. Jarren (Hrsg.), Medien und Journalismus 1. Wiesbaden: Springer. S. 15-30.

Berger, Peter L. \& Luckmann, Thomas (1969). Die gesellschaftliche Konstruktion der Wirklichkeit: Eine Theorie der Wissenssoziologie. Frankfurt a. M.: Fischer.

Bonfadelli, Heinz, Jarren, Otfried \& Siegert, Gabriele (2005). Einfübrung in die Publizistikwissenschaft. Bern: Haupt Verlag.

Burkart, Roland (2002). Kommunikationswissenschaft. Wien/Köln/Weimar: Böhlau.

Charlton, Michael (2001). Produktion und Rezeption von Massenmedien als soziales Handeln. In T. Sutter \& M. Charlton (Hrsg.), Massenkommunikation, Interaktion und soziales Handeln. Wiesbaden: Westdeutscher Verlag. S. 46-66.

Clark, Herbert H. (1996). Using language. Cambridge: Cambridge University Press.

Deppermann, Arnulf (2001). Gespräche analysieren. Opladen: Leske + Budrich.

Deppermann, Arnulf (2008). Verstehen im Gespräch. In H. Kämper \& L. Eichinger (Hrsg.), Sprache - Kognition - Kultur: Sprache zwischen mentaler Struktur und kultureller Prägung. Berlin/New York: de Gruyter. S. 225-261.

Esser, Hartmut (2000). Soziologie - Spezielle Grundlagen: Soziales Handeln (Bd. 3). Frankfurt a. M./New York: Campus.

Fuchs, Peter (1993). Moderne Kommunikation: Zur Theorie des operativen Displacements. Frankfurt a. M.: Suhrkamp.

Geser, Hans (1990). Die kommunikative Mehrebenenstruktur elementarer Interaktionen. Kölner Zeitschrift für Soziologie und Sozialpsychologie, 42, (2), 207-231.

Goffman, Erving (1969). Wir alle spielen Theater: Die Selbstdarstellung im Alltag. München: Piper.

Goffman, Erving (1971 a). Interaktionsrituale: U'ber Verhalten in direkter Kommunikation. Frankfurt a. M.: Suhrkamp.

Goffman, Erving (1971 b). Verhalten in sozialen Situationen: Strukturen und Regeln der Interaktion im öffentlichen Raum. Gütersloh: Bertelsmann.

Goffman, Erving (1974). Das Individuum im öffentlichen Austausch: Mikrostudien zur öffentlichen Ordnung. Frankfurt a. M.: Suhrkamp.

Goffman, Erving (1977). Rahmen-Analyse: Ein Versuch über die Organisation von Alltagserfabrung. Frankfurt a. M.: Suhrkamp.

Goffman, Erving (1981 a). Strategische Interaktion. München: Hanser.

Goffman, Erving (1981 b). Response Cries. In E. Goffman (Hrsg.), Forms of Talk. Philadelphia: University of Pennsylvania Press. S. 78-122. 
Goffman, Erving (2005). Erwiderungen und Reaktionen. In H. Knoblauch, C. Leuenberger \& B. Schnettler (Hrsg.), Erving Goffman. Rede-Weisen: Formen der Kommunikation in sozialen Situationen. Konstanz: UVK. S. 73-150.

Goodwin, Charles (1981). Conversational organisation: Interaction betweeen speakers and hearers. New York: Acadamic Press.

Graumann, Carl Friedrich (1972). Interaktion und Kommunikation. In C.F. Graumann (Hrsg.), Handbuch der Psychologie: Sozialpsychologie (Bd. 7). Göttingen: Verlag für Psychologie. S. 1109-1262.

Habermas, Jürgen (1981). Theorie des kommunikativen Handelns (Bd. 1-2). Frankfurt a. M.: Suhrkamp.

Hahn, Alois (2002). Absichtliche Unabsichtlichkeit. Sozialer Sinn, 3, S. 37-58.

Hausendorf, Heiko (2001). Warum wir im Fernsehen so häufig begrüßt und angeredet werden: Eine exemplarische Studie am Beispiel der Sendung mit der Maus. In T. Sutter \& M. Charlton (Hrsg.), Massenkommunikation, Interaktion und soziales Handeln. Wiesbaden: Westdeutscher Verlag. S. 185-213.

Hausendorf, Heiko (2003). Deixis and speech situation revisited: the mechanism of perceived perception. In F. Lenz (Hrsg.): Deictic conceptualisation of space, time and person. Amsterdam/Philadelphia: John Benjamins. S. 249-269.

Hausendorf, Heiko et al. (2015). Ko-Konstruktionen in der Schrift? Zur Unterscheidung von Face-to-faceInteraktion und Textkommunikation am Beispiel des Editierens. In U. Dausendschön-Gay, E. Gülich \& U. Krafft (Hrsg.), Ko-Konstruktionen in der Interaktion: die gemeinsame Arbeit an Äußerungen und anderen sozialen Ereignissen. Bielefeld: transcript. S. 111-138.

Hebdige, Dick (1998). Stil als absichtliche Kommunikation. In P. Kemper, T. Langhoff \& U. Sonnenschein (Hrsg.), „but I like it": Jugendkultur und Popmusik. Stuttgart: Reclam. S. 392-419.

Horton, Donald \& Wohl, Richard R. (1956). Mass Communication and para-social interaction: Observations on intimacy at a distance. Psychiatry, 19, 215-229.

Jäckel, Michael (1995). Interaktion: Soziologische Anmerkungen zu einem Begriff. Rundfunk und Fernsehen, 43, (4), 463-476.

Jäckel, Michael (2005). Medienwirkungen: Ein Studienbuch zur Einführung. Wiesbaden: VS.

Jefferson, Gail (1972). Side Sequences. In D. Sudnow (Hrsg.), Studies in social interaction. New York: Free Press. S. 294-338.

Joas, Hans (Hrsg.). (2001). Lehrbuch der Soziologie. Frankfurt a. M./New York: Campus.

Kallmeyer, Werner \& Schütze, Fritz (1976). Konversationsanalyse. Studium Linguistik, 1, 1-28.

Keller, Reiner, Reichertz, Jo \& Knoblauch, Hubert (Hrsg.). (2013). Kommunikativer Konstruktivismus: Theoretische und empirische Arbeiten zu einem neuen wissenssoziologischen Ansatz. Wiesbaden: Springer.

Knoblauch, Hubert (1995). Kommunikationskultur: Die kommunikative Konstruktion kultureller Kontexte. Berlin/New York: de Gruyter.

Knoblauch, Hubert (2013). Grundbegriffe und Aufgaben des kommunikativen Konstruktivismus. In R. Keller, J. Reichertz \& H. Knoblauch (Hrsg.), Kommunikativer Konstruktivismus: Theoretische und empirische Arbeiten zu einem neuen wissenssoziologischen Ansatz. Wiesbaden: Springer. S. 25-47.

Linke, Angelika, Nussbaumer, Markus \& Portmann, Paul R. (1996). Studienbuch Linguistik. Tübingen: Niemeyer.

Luckmann, Thomas (1992). Theorie des sozialen Handelns. Berlin/New York: de Gruyter.

Luhmann, Niklas (1984). Soziale Systeme: Grundriss einer allgemeinen Theorie. Frankfurt a. M.: Suhrkamp.

Luhmann, Niklas (1988). Wie ist Bewusstsein an Kommunikation beteiligt? In H.U. Gumbrecht \& L.K. Pfeiffer (Hrsg.), Materialität der Kommunikation. Frankfurt a. M.: Suhrkamp. S. 884-904.

Luhmann, Niklas (1997). Die Gesellschaft der Gesellschaft (Bd. 1-2). Frankfurt a. M.: Suhrkamp.

Mead, George Herbert (1983). Gesammelte Aufsätze (Bd. 2). Frankfurt a. M.: Suhrkamp.

Mead, George-Herbert (1995). Geist, Identität und Gesellschaft. Frankfurt a. M.: Suhrkamp.

Merten, Klaus (1977). Kommunikation: Eine Begriffs- und Prozessanalyse. Opladen: Westdeutscher Verlag. 
Meyrowitz, Joshua (1990 a). Using Contextual Analysis to Bridge the Study of Mediated and Unmediated Behavior. In B.D. Ruben \& L.A. Lievrouw (Hrsg.), Mediation, Information and Communication: Information and Behavior (Bd. 3). New Brunswick: Transaction Press. S. 67-94.

Meyrowitz, Joshua (1990 b). Redefining the Situation: Extending Dramaturgy into a theory of social change and media effects. In S.H. Riggins (Hrsg.), Beyond Goffman: studies on communication, institution, and social interaction. Berlin: de Gruyter. S.65-97.

Mondada, Lorenza \& Schmitt, Reinhold (Hrsg.). (2010). Situationseröffnungen: Zur multimodalen Herstellung fokussierter Interaktion. Tübingen: Narr.

Newcomb, Theodore M. (1953). An Approach of the Study of Communicative Acts. Psychological Review, 60, (6), 393-404.

Newcomb, Theodore M., Turner, Ralph H. \& Converse, Philip E. (1965). Social Psychology: the study of buman interaction. New York: Holt, Rinehart and Winston.

Neuberger, Christoph. (2007). Interaktivität, Interaktion, Internet. Publizistik, 52, (1), 33-50.

Nöth, Winfried (2000). Handbuch der Semiotik. Stuttgart/Weimar: Metzler.

Oevermann, Ulrich, Allert, Tilmannm, Konau, Elisabeth \& Krambeck, Jürgen (1983). Die Methodologie einer „objektiven Hermeneutik“. In P. Zedler \& H. Moser (Hrsg.), Aspekte qualitativer Sozialforschung: Studien zu Aktionsforschung, empirischer Hermeneutik und reflexiver Sozialtechnologie. Opladen: Leske. S. 94-123.

Oevermann, Ulrich (2000). Die Methode der Fallrekonstruktion in der Grundlagenforschung sowie der klinischen und pädagogischen Praxis. In K. Kraimer (Hrsg.), Die Fallrekonstruktion: Sinnverstehen in der sozialwissenschaftlichen Forschung. Frankfurt a. M.: Suhrkamp. S. 58-156.

Posner, Roland (1985). Nonverbale Zeichen in öffentlicher Kommunikation: Zu Geschichte und Gebrauch der Begriffe 'verbal' und 'nonverbal', 'Interaktion' und 'Kommunikation', 'Publikum' und 'Offentlichkeit', 'Medium' und 'Massemedium' und 'multimedial'. Zeitschrift für Semiotik, 7, (3), 235-271.

Rafaeli, Sheizaf (1988). Interactivity: From New Media to Communication. In R.P. Hawkins, J.M. Wiemann \& S. Pingree (Hrsg.), Advancing Communication Science: Merging Mass and Interpersonal Processes. Newbury Park al.: Sage. S. 110-134.

Reichertz, Jo (2009). Kommunikationsmacht. Was ist Kommunikation und was vermag sie? Und weshalb vermag sie das? Wiesbaden: VS.

Reimann, Horst (1968). Kommunikations-Systeme. Tübingen: J.C.B. Mohr.

Rusch, Gebhard (1994). Kommunikation und Verstehen. In K. Merten, S.J. Schmidt \& S. Weichenberg (Hrsg.), Die Wirklichkeit der Medien. Opladen: Westdeutscher Verlag. S.60-78.

Saussure, Ferdinand de (1967). Grundfragen der allgemeinen Sprachwissenschaft. Berlin/New York: de Gruyter.

Scannell, Paddy (1991). Introduction: The Relevance of Talk. In P. Scannell (Hrsg.), Broadcast talk. London: Sage. S. 1-13.

Schegloff, Emanuel A. (1972). Sequencing in conversational openings. In J.J. Gumpertz \& D. Hymes (Hrsg.), Directions in Sociolinguistics: The Ethnography of Speaking. New York: Holt, Rinehart and Winston. S. 346-380.

Schegloff, Emanuel A. (1997). Third Turn Repair. In G.R. Guy, C. Feagin, D. Schiffrin \& J. Baugh (Hrsg.), Towards a Social Science of Language - Papers in honor of William Labov: Social Interaction and Discourse Structures (Bd. 2). Amsterdam: John Benjami. S. 31-40.

Schegloff, Emanuel A. (2007). Sequence organization in interaction. Cambridge: Cambridge University Press.

Schmidt, Axel (2011). Medien | Interaktion: Zum Zusammenhang von Handeln und Darstellen am Beispiel faktualer Fernsehformate. Baden-Baden: Nomos.

Schmidt, Siegfried J. (1994). Kognitive Autonomie und soziale Orientierung: Konstruktivistische Bemerkungen zum Zusammenhang von Kognition, Kommunikation, Medien und Kultur. Frankfurt a. M: Suhrkamp.

Schneider, Wolfgang Ludwig (1994). Die Beobachtung von Kommunikation: Zur kommunikativen Konstruktion sozialen Handelns. Opladen: Westdeutscher Verlag.

Schneider, Wolfgang Ludwig (2002). Grundlagen der soziologischen Theorie: Garfinkel-RC-Habermas - Lubmann (Bd. 2). Wiesbaden: Westdeutscher Verlag. 
Schneider, Wolfgang Ludwig (2004). Grundlagen der soziologischen Theorie: Sinnverstehen und Intersubjektivität - Hermeneutik, funktionale Analyse, Konversationsanalyse und Systemtheorie (Bd. 3). Wiesbaden: VS.

Schneider, Wolfgang Ludwig (2005). Grundlagen der soziologischen Theorie: Weber - Parsons - Mead Schütz (Bd. 1). Wiesbaden: VS.

Schütz, Alfred (1974). Der sinnhafte Aufbau der sozialen Welt: Eine Einleitung in die verstehende Soziologie. Frankfurt a. M.: Suhrkamp.

Schütz, Alfred (2003). Symbol, Wirklichkeit und Gesellschaft. In H. Knoblauch, K. Ronald, H. Soeffner \& R. Grathoff (Hrsg.), Alfred Schütz Werkausgabe - Theorie der Lebenswelt 2: Die kommunikative Ordnung der Lebenswelt (Bd. V.2). Konstanz: UVK. S. 117-201.

Schütz, Alfred \& Luckmann, Thomas (1984). Strukturen der Lebenswelt (Bd. 2). Frankfurt a. M.: Suhrkamp.

Schützeichel, Rainer (2004). Soziologische Kommunikationstheorien. Konstanz: UVK.

Schulze, Gerhard (1995). Die Erlebnisgesellschaft: Kultursoziologie der Gegenwart. Frankfurt/New York: Campus.

Shannon, Claude E. \& Weaver, Warren (1949). The mathematical theory of communication. Urbana: University of Illinois Press.

Simmel, Georg (1995). Soziologie: Untersuchung über die Formen der Vergesellschaftung. Frankfurt a. M.: Suhrkamp.

Thompson, John B. (1995). The media and modernity: A social theory of the media. Cambridge et al.: Polity Press.

Ungeheuer, Gerold (1987). Kommunikationstheoretische Schriften 1. Aachen: Rader.

Watzlawick, Paul, Beavin, Janet H. \& Jackson, Don D. (1969). Menschliche Kommunikation: Formen, Störungen, Paradoxien. Bern/Stuttgart: Huber.

Weber, Max (1980). Wirtschaft und Gesellschaft: Grundriß der verstehenden Soziologie. Tübingen: Mohr.

Willems, Herbert (1997). Habitus und Rabmen: Zum theoretischen und methodischen Ansatz Erving Goffmans - Vergleiche, Anschlüsse und Anwendungen. Frankfurt a. M: Suhrkamp.

Willems, Herbert (2012). Synthetische Soziologie: Idee, Entwurf und Programm. Wiesbaden: VS.

Wilson, Thomas P. (1973). Theorie der Interaktion und Modelle soziologischer Erklärung. In AG Bielefelder Soziologen (Hrsg.), Alltagswissen, Interaktion und gesellschaftliche Wirklichkeit (Bd. 1). Reinbek: Rowohlt. S. 54-79.

Wulff, Hans J. (1993). Phatische Gemeinschaft/Phatische Funktion: Leitkonzepte einer pragmatischen Theorie des Fernsehens. Montage AV, 2, (1), 142-163. 